\title{
Wavelet Kernel Least Squares Twin Support Vector Regression for Wind Speed Prediction
}

\section{Barenya Bikash Hazarika}

NIT Arunachal Pradesh: National Institute of Technology Arunachal Pradesh

\section{Deepak Gupta ( $\nabla$ deepak@nitap.ac.in )}

NIT Arunachal Pradesh: National Institute of Technology Arunachal Pradesh https://orcid.org/00000002-6375-8615

\section{Narayanan Natarajan}

Dr Mahalingam College of Engineering and Technology

\section{Research Article}

Keywords: Wind speed prediction, wavelet, kernel, twin support vector regression, primal least squares

Posted Date: August 17th, 2021

DOI: https://doi.org/10.21203/rs.3.rs-522218/v1

License: @ (i) This work is licensed under a Creative Commons Attribution 4.0 International License. Read Full License

Version of Record: A version of this preprint was published at Environmental Science and Pollution Research on January 24th, 2022. See the published version at https://doi.org/10.1007/s11356-02218655-8. 


\title{
Wavelet kernel least squares twin support vector regression for wind speed prediction
}

\author{
Barenya Bikash Hazarika', Deepak Gupta², Narayanan Natarajan ${ }^{3}$ \\ ${ }^{1,2}$ Department of Computer Science and Engineering, National Institute of Technology \\ Arunachal Pradesh, Yupia, Papum Pare - 791112, Arunachal Pradesh, India. \\ ${ }^{3}$ Department of Civil Engineering, Dr. Mahalingam College of Engineering and Technology, \\ Pollachi - 642003, Tamil Nadu, India.
}

Abstract:Wind energy is a potent yet freely available renewable energy. It is essential to estimate the wind speed (WS)precisely to makeaprecise estimation of wind power at wind power generating stations.Generally, the WS data is non-stationary. Wavelets have the potential to deal with the non-stationarilyindatasets. On the other hand, the prediction ability of primal least square support vector regression (PLSTSVR) has never been tested to best of our knowledge for WS prediction. Hence, in this work, wavelet kernel-based LSTSVR models are proposed for WS prediction. They are Morlet wavelet kernel LSTSVR and Mexican Hat wavelet kernel LSTSVR.HourlyWS data are collected from four different stations namely Chennai, Madurai, Salem and Tirunelveli in Tamil Nadu, India. The performance of the proposed models isevaluated using root mean square, mean absolute, symmetric mean absolute percentage, mean absolute scaled error and $\mathrm{R}^{2}$. The results of the proposed models are compared with twin support vector regression (TSVR), PLSTSVR and large-margin distribution machine-based regression (LDMR). Based on the results of the performance indicators, the performance of the proposed models is better when compared to other models.

Keywords:Wind speed prediction; wavelet;kernel; twin support vector regression;primal least squares

\footnotetext{
${ }^{1}$ barenya1431@gmail.com

${ }^{2}$ Corresponding author: deepak@nitap.ac.in

3itsrajan2002@yahoo.co.in
} 


\section{Introduction}

Renewable energy sources are progressively made use of because of the amazingly booming contamination degrees in the air, water, and soil. Wind energy has ended up being the prime focus for energy developers as a result of the accessibility of megawatt size wind equipment, environmental friendliness, low cost of maintenance, ease of availability, etc. Moreover, wind resource is a tidy, limitless and free resource. This resource has offered mankind for numerous centuries in driving the wind turbines and pumping water (Bakhsh et al. 1985). To utilize this resource efficiently, prediction of WS plays a critical role. This is necessary for site planning, performance analysis, and deciding the optimal size of the wind turbine. Rehman and Halawani (1994) predicted wind speed using the autoregressive moving average method. Recently, machine learning (ML) models such as Artificial Neural Network (ANN), Support Vector Machine (SVM), Gaussian Process Regression (GPR), Fuzzy Logic (FL), and Extreme Learning Machine (ELM) has been extensivelyused for this purpose. Selection of the most appropriate ML technique is very important for obtaining accurate results. Apart from these ML techniques, researchers are nowadays focused on the development of hybrid ML tools to achieve accurate WS forecasting. Furthermore, hybrid ML has the advantage of both algorithms.

Salcedo-Sanzet al. (2011) tried to estimate the short wind speed using an evolutionary SVR model. Wang et al. (2015) forecasted the WS using support vector regression (SVR) optimized by the cuckoo search optimization algorithm. Wang et al. (2016) utilized the SVM whose parameter search was optimized using a novel Steepest descent Cuckoo search algorithm for the dataset which was pre-processed using the empirical mode decomposition. Khosravi et al. (2018) forecasted the WS and wind direction with the help of multi-layer feed-forward ANN, SVM with radial basis function(RBF) and adaptive neuro-fuzzy inference system (ANFIS) optimized using a swarm optimization (SO) called particleSOalgorithm. Mi et al. (2019) developed a new WS multi-step forecastingframework based on the singular spectrum analysis, empirical mode decomposition and convolution SVM. Wu and Lin (2019) forecasted the WS based on LS-SVM optimized using the bat optimization algorithm. They applied variation mode decomposition to disintegrate the original WS series into separate sub-serieswithdifferent frequencies. Fu et al. (2019) coupled SVM with improved chicken SO algorithm for the prediction of WS and the results were compared with SVM-CSO. Xiang et al. (2019) forecasted WS based on the hybrid of improved empirical wavelet transform and LS-SVM. Li et al. (2020) 
57 performed wind power prediction based on SVM with improved dragonfly algorithm. Kumar 58 and Deeba (2020) used a long short-term memory network model and variants of the SVMs for 59 forecasting the WS in the neighbourhood of windmills.

In addition to the above discussed hybrid SVM models, wavelet transform has been used in combination with SVM for the forecasting the WS of a location.There are two types of studies where the wavelet transform (WT) has been adopted. In some of the earlier studies, wavelet has been adopted for the processing of the WS data by considering the same as a signal, while in some other cases a wavelet-based kernel has been used in the SVM model. Some of the studies which fall under the former category have been performed by Liu et al. (2014), Sangita and Deshmukh (2011), Sun et al.(2013), Sivanagaraja et al. (2014), and Prasetyowati et al. (2019). While most of them have considered the only SVM for the former case, Dhiman et al. (2019) have adopted not only SVM but also its variants. Theyanalyzed the efficiency of a hybrid model consisting of WT and several variants of SVR like $\varepsilon$-SVR, Least squares SVR, Twin SVR (TSVR) and $\varepsilon$-TSVR for the prediction of WS at a farm in Spain.Only a few studies about the latter case have been performed for WS prediction. Zeng et al. (2012) performed wind power prediction using a novel wavelet SVM that changes between the RBF kernel and a Mexican hat kernel. They established that the wavelet SVM outperforms the radial basis function kernel and Mexican hat kernels. He and $\mathrm{Xu}$ (2019) predicted the WS of Ningxia using a combination of a wavelet as well as polynomial kernel function and concluded that the combined kernel has better accuracy compared to the single kernel function. Few very recent prominent works are portrayed in Table 1.

Table 1: List of a few recent contributions for wind speed prediction.

\begin{tabular}{|c|c|c|c|c|}
\hline $\begin{array}{c}\text { Sl } \\
\text { No. }\end{array}$ & Reference & Model/Models proposed & $\begin{array}{c}\text { Country/ } \\
\text { Coutries }\end{array}$ & Term \\
\hline 1 & Mi et al. (2019) & $\begin{array}{c}\text { Singular spectrum analysis, empirical mode } \\
\text { decomposition and convolution neural network-SVM }\end{array}$ & China & Short- term \\
\hline 2 & Zhang et al. (2019) & $\begin{array}{c}\text { LSTM and Gaussian process regression (GPR) } \\
\text { China }\end{array}$ & Short- term \\
\hline 3 & Tian et al. (2019) & $\begin{array}{c}\text { Improved PSO optimized error minimized extreme } \\
\text { learning machine (EM-ELM) }\end{array}$ & China & Short-term \\
\hline 4 & $\begin{array}{c}\text { Jamil and Zeeshan } \\
(2019)\end{array}$ & ANN based chaotic approach & India & $\begin{array}{c}\text { Short-term and Long- } \\
\text { term }\end{array}$ \\
\hline 5 & Qolipour et al. (2019) & Grey-ELM & Iran & Long- term \\
\hline 6 & $\begin{array}{c}\text { Jha and Bilalovikj } \\
(2019)\end{array}$ & ANN & $\begin{array}{c}\text { Germany and } \\
\text { Netherlands }\end{array}$ & Very short- term \\
\hline 7 & $\begin{array}{c}\text { Harboola and Coors } \\
(2019)\end{array}$ & $\begin{array}{c}\text { Short- term } \\
(2020)\end{array}$ & ANN and chaotic approach & $\begin{array}{c}\text { Short-term and Long- } \\
\text { term }\end{array}$ \\
\hline 8
\end{tabular}




\begin{tabular}{|c|c|c|c|c|}
\hline 9 & $\begin{array}{l}\text { Vinothkumar and } \\
\text { Deeba }(2020)\end{array}$ & LSTM and SVM & India & Short-term \\
\hline 10 & Zhang et al. (2020) & GA-ANN & China & Short- term \\
\hline 11 & Tian (2020) & $\begin{array}{c}\text { Local mean decomposition and Improved FA } \\
\text { optimized least squares SVM }\end{array}$ & China & Short- term \\
\hline 12 & $\begin{array}{l}\text { Blanchard and } \\
\text { Samanta (2020) }\end{array}$ & ANN & USA & Short- term \\
\hline 14 & $\begin{array}{l}\text { Biswas and Sinha } \\
(2020) \\
\end{array}$ & $\begin{array}{l}\text { Singular spectrum analysis, SVM and variational } \\
\text { mode decomposition }\end{array}$ & India & Short-term \\
\hline 15 & Liu et al. (2020) & Jaya-SVM & China & Short-term \\
\hline 16 & $\begin{array}{l}\text { Ruiz-Aguilar et al. } \\
\text { (2021) }\end{array}$ & EMD-ANN & Spain & $\begin{array}{l}\text { Short-term and Long- } \\
\text { term }\end{array}$ \\
\hline 17 & Xiao et al. (2021) & Self adaptive kernel ELM & China & Short-term \\
\hline 18 & $\begin{array}{l}\text { Wanag and Yang } \\
(2021)\end{array}$ & Optimized Elman Neural Network & China & Very short- term \\
\hline
\end{tabular}

In this study, the main contributions are:

1. Motivated by the contribution of Zhang et al. (2004) and Ding et al. (2014), two novel wavelet-based models, namely, Morlet wavelet kernel LSTSVR(MKLSTSVR) and Mexican Hat LSTSVR (MHKLSTSVR) models are proposed for WS prediction.

2. Inspired by the PLSTSVR model, the optimization problem of the proposed MKLSTSVR and MHKLSTSVR are solved in primal, which always shows better performance compared to solving them in duals (Gupta 2017).

3. To expand the kernel selection range of the PLSTSVR model and to improve its generalization ability, the wavelet kernels are embedded in the PLSTSVR model.

The models have been applied to WS data of four locations in the state of Tamil Nadu, India and the performance of these models has been compared with other ML models such as TSVR, primal least squares twin support vector regression (PLSTSVR) and large-margin distribution machine-based regression (LDMR). The rest of this paper is organized as follows: Section 2 briefly describe the related works. Section 3 describes the wavelet analysis briefly.

Section 3 proposes the two novel models. Dataset description and area of study is presented in Section 4. Experimental and numerical analyses are presented in Section 5 while Section 6shows the conclusion.

\section{Related works}

Consider $m$ is the total number of samples. The total training samplescan be represented as $\left\{x_{i}\right\}_{i=1}^{m} \in R^{n} . x_{i} \in R$ and $y_{i} \in R^{N}$ indicates the input training points and original output 
respectively. Also, let us consider $A \in R^{m \times n}$ are the training datapoints. Here the $i^{t h}$ row vector can be represented as $x_{i}^{t} . y=\left(y_{1, \ldots} y_{m}\right)$ denotes the observed datapoints.

\subsection{The TSVR model}

TSVR (Peng, 2010)seeks for two non-parallel functions termed as $\varepsilon$ - insensitive downbound $f_{1}(x)=K\left(x^{t}, D^{t}\right) w_{1}+b_{1}$ and up-bound function $f_{2}(x)=K\left(x^{t}, D^{t}\right) w_{2}+b_{2}$, respectively. The primal problems of TSVR are expressed as:

$$
\begin{aligned}
& \min \frac{1}{2}\left\|y-\varepsilon_{1} e-\left(K\left(G, G^{t}\right) w_{1}+b_{1} e\right)\right\|^{2}+C_{1} e^{t} \psi_{1}, \\
& \text { s.t., } y-\left(K\left(G, G^{t}\right) w_{1}+b_{1} e\right) \geq \varepsilon_{1} e-\psi_{1}, \psi_{1} \geq 0
\end{aligned}
$$

and

$$
\min \frac{1}{2}\left\|y-\varepsilon_{2} e-\left(K\left(G, G^{t}\right) w_{2}+b_{2} e\right)\right\|^{2}+C_{2} e^{t} \psi_{2},
$$

$$
\text { s.t., } y-\left(K\left(G, G^{t}\right) w_{2}+b_{2} e\right) \geq \varepsilon_{2} e-\psi_{2}, \psi_{2} \geq 0 \text {. }
$$

where regularization parameters are $C_{1}, C_{2}>0$, and input parameters are $\varepsilon_{1}, \varepsilon_{2}>0$; the slack variables are indicated by $\psi_{1}$ and $\psi_{2}$.

By introducing the Lagrangian multipliers $\gamma_{1}, \gamma_{2}>0$ and apply the K.K.T. sufficient conditions on (2), the duals of (1) and (2) are expressed as:

$$
\max -\frac{1}{2} \gamma_{1}^{t} M_{1}\left(M_{1}^{t} M_{1}\right)^{-1} M_{1}^{t} \gamma_{1}+R^{t} M_{1}\left(M_{1}^{t} M_{1}\right)^{-1} M_{1}^{t} \gamma_{1}-R^{t} \gamma_{1}
$$

s.t., $0 \leq \gamma_{1} \leq C_{1} e$

and

$$
\max -\frac{1}{2} \gamma_{2}^{t} M_{1}\left(M_{1}^{t} M_{1}\right)^{-1} M_{1}^{t} \gamma_{2}+Q^{t} M_{1}\left(M_{1}^{t} M_{1}\right)^{-1} M_{1}^{t} \gamma_{2}-Q^{t} \gamma_{2},
$$

120

s.t., $0 \leq \gamma_{2} \leq C_{2} e$,

121 determined as: 


$$
\left[\begin{array}{l}
w_{1} \\
b_{1}
\end{array}\right]=\left(M_{1}^{t} M_{1}+\partial I\right)^{-1} M_{1}^{t}\left(R-\gamma_{1}\right)
$$

123 and

124

$$
\left[\begin{array}{l}
w_{2} \\
b_{2}
\end{array}\right]=\left(M_{1}^{t} M_{1}+\partial I\right)^{-1} M_{1}^{t}\left(Q+\gamma_{2}\right),
$$

125 where $\partial>0$ and $I$ is an identity matrix.

126

127

128

129

130

131

132

133

134

135

136

137

138

139

140 and

141

and

\subsection{The PLSTSVR model}

In order to diminish the computational complexity of the TSVR model, Huang et al. (2013) suggested a novel LSTVSR solved in primal termed as least PLSTSVR. The formulation of PLSTSVR can be expressed as:

$$
\min \frac{1}{2}\left\|R-\left(K\left(G, G^{t}\right) w_{1}+b_{1} e\right)\right\|^{2}+\frac{1}{2} C_{1} \psi_{1}^{t} \psi_{1}
$$

$$
\text { s.t., } y-\left(K\left(G, G^{t}\right) w_{1}+b_{1} e\right)=\varepsilon_{1} e-\psi_{1}
$$

$$
\begin{aligned}
& \min \frac{1}{2}\left\|Q-\left(K\left(G, G^{t}\right) w_{2}+b_{2} e\right)\right\|^{2}+\frac{1}{2} C_{2} \psi_{2}{ }^{t} \psi_{2}, \\
& \text { s.t., }\left(K\left(G, G^{t}\right) w_{2}+b_{2} e\right)-y=\varepsilon_{2} e-\psi_{2},
\end{aligned}
$$

where $R=\left(Y-\varepsilon_{1} e\right)$ and $Q=\left(y+\varepsilon_{2} e\right)$.

Now, by putting the values of (5) and (6)the slack vectors in the objective functions, and further compute the gradient with respect to $w_{i}$ and $b_{i}$ for $i=1,2$, and equate to zero; we get,

$$
\left[\begin{array}{l}
w_{1} \\
b_{1}
\end{array}\right]=\left(M_{1}^{t} M_{1}+\partial I\right)^{-1} M_{1}^{t} R
$$

$$
\left[\begin{array}{l}
w_{2} \\
b_{2}
\end{array}\right]=\left(M_{1}^{t} M_{1}+\partial I\right)^{-1} M_{1}^{t} Q,
$$


142 where $M_{1}=\left[K\left(G, G^{t}\right) \quad e\right]$ is the augmented matrix; $\partial>0$ is a small positive integer and $I$ is an 143 identity matrix.

144 The final regressor of PLSTSVRfor any new instance $x \in R^{n}$ can be calculated as:

$$
f(x)=\frac{1}{2}\left(K\left(x^{t}, G\right)\left(w_{1}+w_{2}\right)+\left(b_{1}+b_{2}\right)\right) .
$$

\subsection{The LDMR model}

Recently, Rastogi et al.(2020)introduced a margin distribution-based LDMRwhich was on the spiritof the LDM model (Zhang and Zhou 2014). LDMR simultaneously minimizes the $\varepsilon$ - insensitive loss functionand the quadratic loss function. Hence, LDMR shows improved regression performance for noisy data and it considers the minimization of scattering which is inside the $\varepsilon$-tube. The primal problem of LDMR can be expressed as

$$
\min \frac{l_{2}}{2}\|y-(G w+e b)\|^{2}+\frac{1}{2} C e^{t}\left(\psi_{1}+\psi_{2}\right)+\frac{l_{1}}{2}\|w\|^{2}
$$

$$
\text { s.t., } y-(G w+e b) \leq e \varepsilon+\psi_{1}, \psi_{1} \geq 0 \text {, }
$$

$$
(G w+e b)-y \leq e \varepsilon+\psi_{2}, \psi_{2} \geq 0,
$$

155 where the input parameters are $\varepsilon, l_{1}, l_{2}, C>0 ;\|w\|^{2}=u_{2}{ }^{t} I_{0} u_{2}$ where $I_{0}=\left[\begin{array}{cc}I & 0 \\ & \cdot \\ & \cdot \\ 0 & \ldots 0\end{array}\right] ; I^{m \times m}$ is an 156 identity matrix.

157 The dual problem of (9) maybeobtained after adding the Lagrangian multiplier and applying the 158 KKT condition as:

$$
\min \frac{1}{2}\left(\gamma_{1}-\gamma_{2}\right)^{t} M_{1}\left(l_{1} I_{0}+l_{2} M_{1}^{t} M_{1}\right)^{-1} M_{1}^{t}\left(\gamma_{1}-\beta_{2}\right)+y^{t} M_{1}\left(l_{1} I_{0}+l_{2} M_{1}^{t} M_{1}\right)^{-1} M_{1}^{t}\left(\gamma_{1}-\gamma_{2}\right)
$$

$$
-y^{t}\left(\gamma_{1}-\gamma_{2}\right)+\varepsilon e^{t}\left(\gamma_{1}+\gamma_{2}\right),
$$

$$
\text { s.t., } 0 \leq \gamma_{1} \leq C e, 0 \leq \gamma_{2} \leq C e \text {, }
$$


After determining the solution from (10) for $\beta_{1}$ and $\beta_{2}$, the unknowns $w$ and $b$ can be obtained as (Hazarika et al. 2020a):

$$
\left[\begin{array}{l}
w \\
b
\end{array}\right]=\left(l_{1} I_{0}+l_{2} M_{1}^{t} M_{1}\right)^{-1} M_{1}^{t}\left(\gamma_{1}-\gamma_{2}+y\right) .
$$

The LDMR regressor $f\left(\right.$.) can be obtained for any new sample $x \in R^{n}$ as:

$$
f(x)=\left[K\left(x^{t}, G^{t}\right) 1\right]\left[\begin{array}{l}
w \\
b
\end{array}\right] .
$$

\section{Proposed Models}

The wavelet transform (WT) is an enhanced version of the conventional Fourier transform (FT) that was introduced by Jean Morlet in 1982 (Morlet et al. 1982a; Morlet et al. 1982b). Recently, the WT analysis has gained a lot of interest among the researchers as it overcomes the two key disadvantages of FT (Sifuzzaman et al. 2009; Hazarika et al. 2020a, Hazarika et al. 2020b):

1) FT is suitable only for stationary signals whereas WT is suitable for both stationary as well as non-stationary datasets.

2) In FT the time information is lost while transforming the time domain to the frequency domain whereas, in WT, the time information is not lost.

Wavelet analysis was initially suggested to enhance seismic signal analysis by switching from short-term analysis of Fourier to improved algorithms to identifyand analyze abrupt signal changes (Daubechies 1990; Daubechies 1992; Mallat 1999). The wavelet has the multiresolution and localization capacity in both, time-domain (TD) and frequency domain (FD). Wavelet transform's TD properties can be elaborated through the wavelet functions which are translated from a wavelet base function (Holland 1992).

The wavelet functions can be derived from the mother wavelet (MW). Let $\lambda(z)$ be the MW function (Hazarika and Gupta 2020). The wavelet function $\lambda(z)$ can be obtained by the temporal translation $t$ and with dilation $\delta$ as:

$$
f_{t}(\delta, t)=\frac{1}{\sqrt{\delta}} \int_{-\infty}^{\infty} t(z) \lambda\left(\frac{z-t}{\delta}\right) \delta z \quad \delta>0 .
$$


Equation (11) can be represented in the time domain as:

$$
f_{t}(\delta, t)=\frac{1}{\sqrt{\delta}} \int_{-\infty}^{\infty} t(\omega) \lambda(a \omega) e^{i \omega} \delta z \quad \delta>0 .
$$

190

From (11) and (12) one can observe that the local characteristics of a signal can be reflected through the wavelet transform. Hence the wavelet transforms as an analytical technique has shown great potential (Zhang et al. 2004; Zhou and Ye, 2006; Ding et al. 2016).

\subsection{Translation-invariant kernel}

The kernel of translation-invariant (TI) is acceptable if and only if the FT is always positive.

Lemmal: Let $\lambda(z)$ be an MW, and let $\delta$ and $t$ denote the dilation and translation. If $z, z^{\prime} \in R^{N}$, then the dot-product wavelet kernel may be expressed as:

$$
K\left(z, z^{\prime}\right)=\prod_{i=1}^{N} h\left(\frac{z_{i}-t_{i}}{\delta}\right) h\left(\frac{z_{i}^{\prime}-t_{i}^{i}}{\delta}\right) .
$$

The TI kernel of (13) may be expressed as

$$
K\left(z, z^{\prime}\right)=\prod_{i=1}^{N} h\left(\frac{z_{i}-z_{i}^{\prime}}{\delta}\right) .
$$

In (14) $N$ represents the total quantity of the sample (Ding et al. 2014).

To generate the TI kernel function, this work uses two wavelet kernel function. They are:

$$
\text { a. Morlet wavelet kernel: }\left(\lambda(z)=\cos (1.75 z) \exp \left(-\frac{z^{2}}{2}\right)\right) \text { and }
$$

b. Mexican Hat wavelet kernel: $\left(\lambda(z)=\left(1-z^{2}\right) \exp \left(-\frac{z^{2}}{2}\right)\right)$.

\subsection{Proposed MKLSTSVR}

MKLSTSVR indicates the PLSTSVR embedded with theMorlet wavelet kernel instead of using the traditional kernels. To be an acceptable kernel the Morlet kernel should satisfy the TI kernel theorem as shown in equation (14).

Lemma 2: The Morlet wavelet kernel function (MK) satisfies the TI kernel theorem as:

$$
K\left(z, z^{\prime}\right)=\prod_{i=1}^{N}\left(\cos \left(1.75 \times \frac{z_{i}-z_{i}^{\prime}}{\delta}\right) \exp \left(-\frac{\left\|z_{i}-z_{i}^{\prime}\right\|^{2}}{2 \delta^{2}}\right)\right)
$$


211 which is an admissible kernel (Zhang et al. 2004). Hence, it is a kernel by which the wavelet

212 kernel trick can be used to construct MKLSTSVR. The basic goal of MKLSTSVR is to search

213 for the optimum wavelet coefficients in feature space spanned by multidimensional wavelet basis

214 (MWB). Thus it obtains the optimal prediction function. Thereby the prediction function for 215 MKLSTSVR can be obtained as:

$$
f(z)=\frac{1}{2} \prod_{i=1}^{N}\left(\cos \left(1.75 \times \frac{z_{i}-z_{i}^{\prime}}{\delta}\right) \exp \left(-\frac{\left\|z_{i}-z_{i}^{\prime}\right\|^{2}}{2 \delta^{2}}\right)\right)\left(w_{1}+w_{2}\right)+\frac{1}{2}\left(b_{1}+b_{2}\right) .
$$

217

218

219

220

221

222

223

224

225

226

227

228

229

230

231

232

233

234

235

236

\subsection{Proposed MHKLSTSVR}

MHKLSTSVR indicates the PLSTSVR combined with the Mexican Hat wavelet kernel rather than the traditional kernels. To be an admissible kernel function, the Mexican Hat kernel should also satisfy the TI kernel theorem as shown in equation (14).

Lemma 3: The Mexican hat wavelet kernel function (MHK) follows the TI kernel theorem as:

$$
K\left(z, z^{\prime}\right)=\prod_{i=1}^{N}\left(1-\left(\frac{z_{i}-z_{i}^{\prime}}{\delta}\right)^{2}\right) \exp \left(-\frac{1}{2}\left(\frac{z_{i}-z_{i}^{\prime}}{\delta}\right)^{2}\right),
$$

which also can be considered as an admissible kernel (Zhang et al. 2004). Therefore, the Mexican Hat wavelet kernel trick can be used to construct MHKLSTSVR. Similar to MKLSTSVR, the basic goal of MHKLSTSVR is to find the optimum wavelet coefficients in feature space spanned by MWB. Thereby it obtains the optimum predictor. Thereby the predictor for MHKLSTSVR can be obtained as:

$$
f(z)=\frac{1}{2} \prod_{i=1}^{N}\left(1-\left(\frac{z_{i}-z_{i}^{\prime}}{\delta}\right)^{2}\right) \exp \left(-\frac{1}{2}\left(\frac{z_{i}-z_{i}^{\prime}}{\delta}\right)^{2}\right)\left(w_{1}+w_{2}\right)+\frac{1}{2}\left(b_{1}+b_{2}\right) .
$$

\section{Dataset Description}

The state of Tamil Nadu is situated in the southern part of India. Tamil Nadu is bordered by the Eastern Ghats in the north, Anaimalai hills and Kerala in the west, Bay of Bengal in the east, Gulf of Mannar and Palk Strait in the southeast, and the Indian Ocean in the south. The climate of Tamil Nadu ranges from sub-humid to semi-arid. The sites selected for this study has been provided in Figure 1. The geographical information such as latitude (LAT), longitude (LON) and altitude (ALT) of the chosen sites are provided in Table 2. Hourly averageWS data recorded at a height of 50m above ground level wascollected from MERRA -2 analyis database 
(NASA) for the period of Jan 1980 to May 2018. However,in our experiments,we have considered the data from Jan 2014 to May 2018.

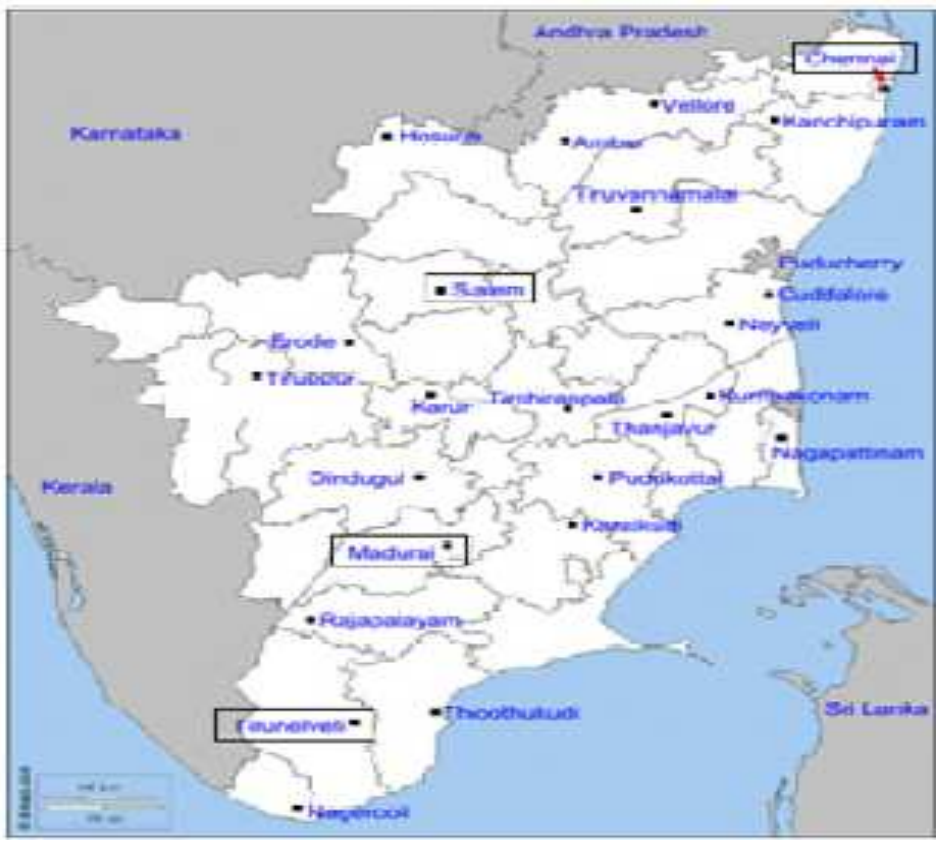

Figure 1.Map of Tamil Nadu showing the chosen four sites (marked with boxes)

Table 2. Geographical locations of the cities in Tamil Nadu, India

\begin{tabular}{clccc}
\hline S.No & Station & $\begin{array}{c}\text { LAT } \\
\left({ }^{\circ} \mathbf{N}\right)\end{array}$ & $\begin{array}{c}\text { LON } \\
\left({ }^{\circ} \mathbf{E}\right)\end{array}$ & $\begin{array}{c}\text { ALT } \\
(\mathbf{m})\end{array}$ \\
\hline 1 & Chennai & 13.08 & 80.27 & 13 \\
\hline 2 & Madurai & 9.92 & 78.11 & 137 \\
\hline 3 & Salem & 11.66 & 78.14 & 286 \\
\hline 4 & Tirunelveli & 8.71 & 77.75 & 41 \\
\hline
\end{tabular}

The statisticsof the WS data of the above four stations has been provided in Table 3 below.

Table 3. Statistics of the wind speed data

\begin{tabular}{clcccc}
\hline S.No & Station & $\begin{array}{c}\text { Mean wind } \\
\text { speed }(\mathbf{m} / \mathbf{s})\end{array}$ & $\begin{array}{c}\text { Median } \\
(\mathbf{m} / \mathbf{s})\end{array}$ & $\begin{array}{c}\text { Maximum } \\
\text { wind speed } \\
(\mathbf{m} / \mathbf{s})\end{array}$ & $\begin{array}{c}\text { Standard } \\
\text { deviation } \\
(\mathbf{m} / \mathbf{s})\end{array}$ \\
\hline 1 & Chennai & 6.228 & 6.38 & 18.1 & 2.381 \\
\hline 2 & Madurai & 4.522 & 4.5 & 13.86 & 1.916 \\
\hline 3 & Salem & 4.619 & 4.5 & 14.43 & 2.049 \\
\hline 4 & Tirunelveli & 5.468 & 5.41 & 22.64 & 2.377 \\
\hline
\end{tabular}

The maximum wind speed of $22.64 \mathrm{~m} / \mathrm{s}$ is noticed in Tirunelveli and the lowest wind speed of $13.86 \mathrm{~m} / \mathrm{s}$ is noticed in Madurai. The observation is similar for the mean WS too. Figure 2 shows the hourly wind speed data from January 2014 to May 2018. 


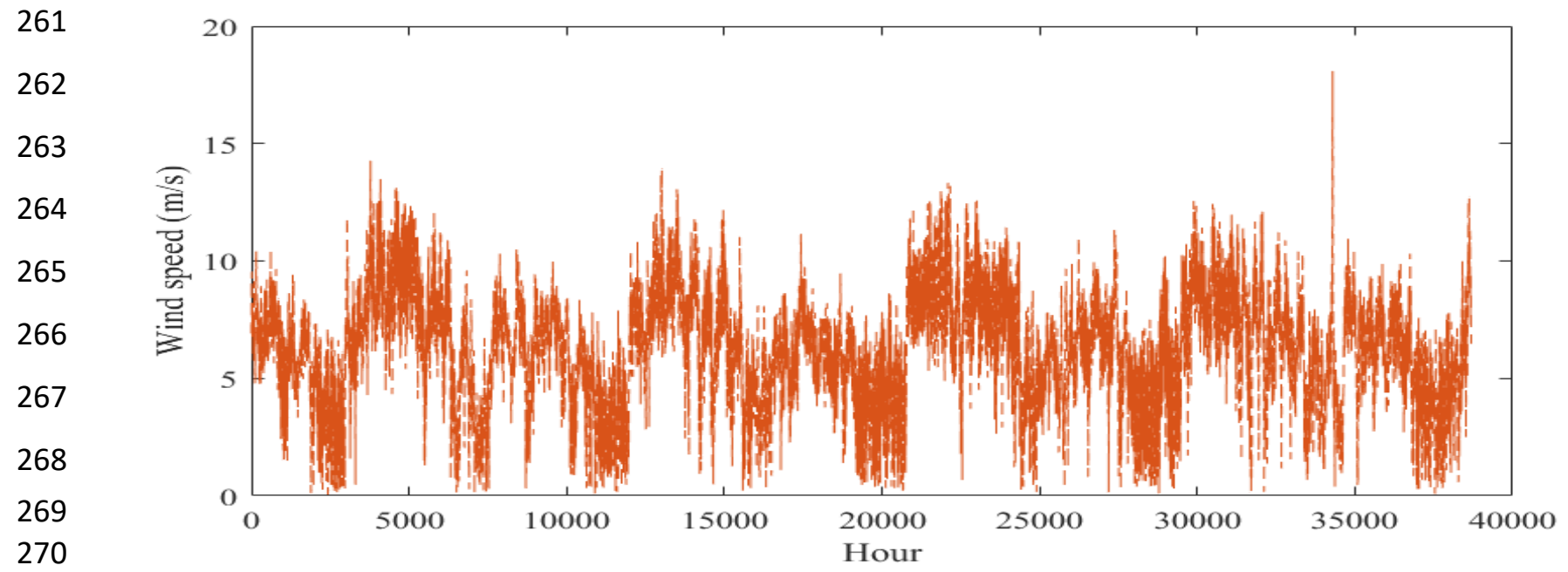

271

(a) Chennai Station

272

273

274

275

276

277

278

279

280

281

282

283

284

285

286

287

288

289

290

291

292

293

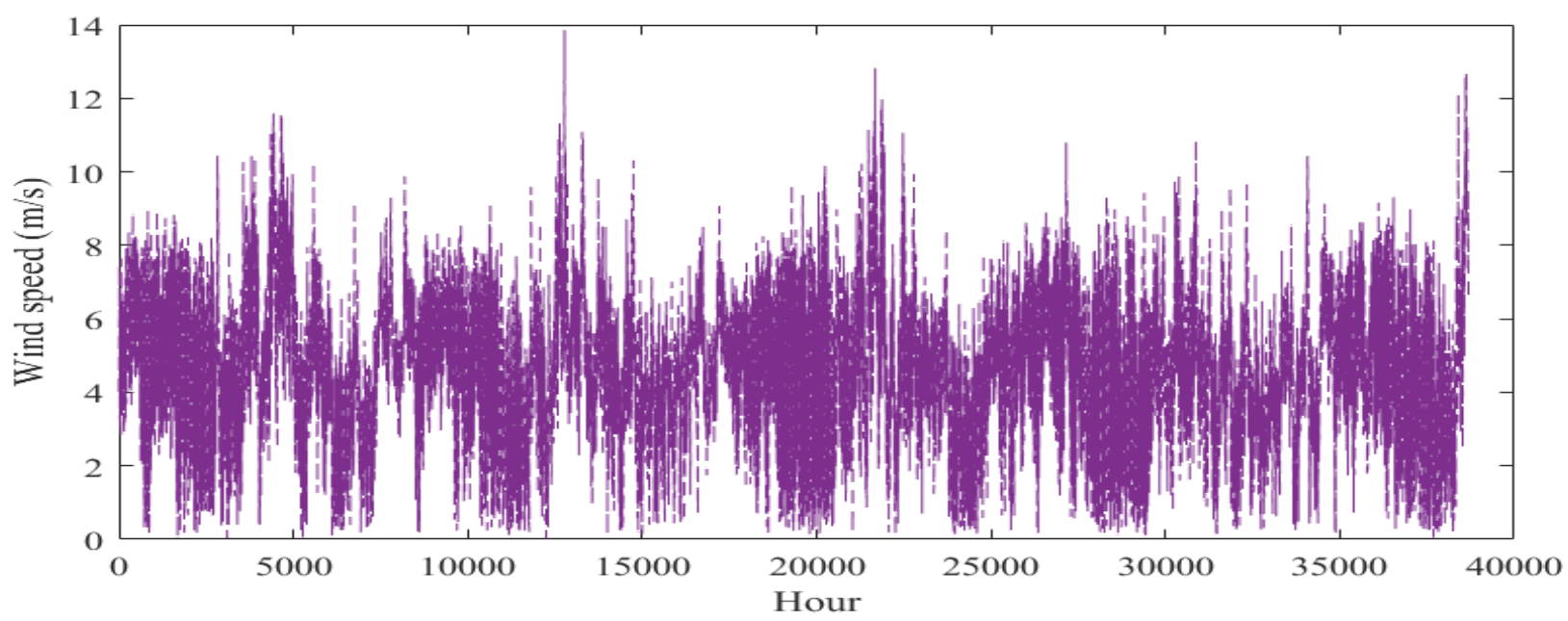

(b) Madurai Station

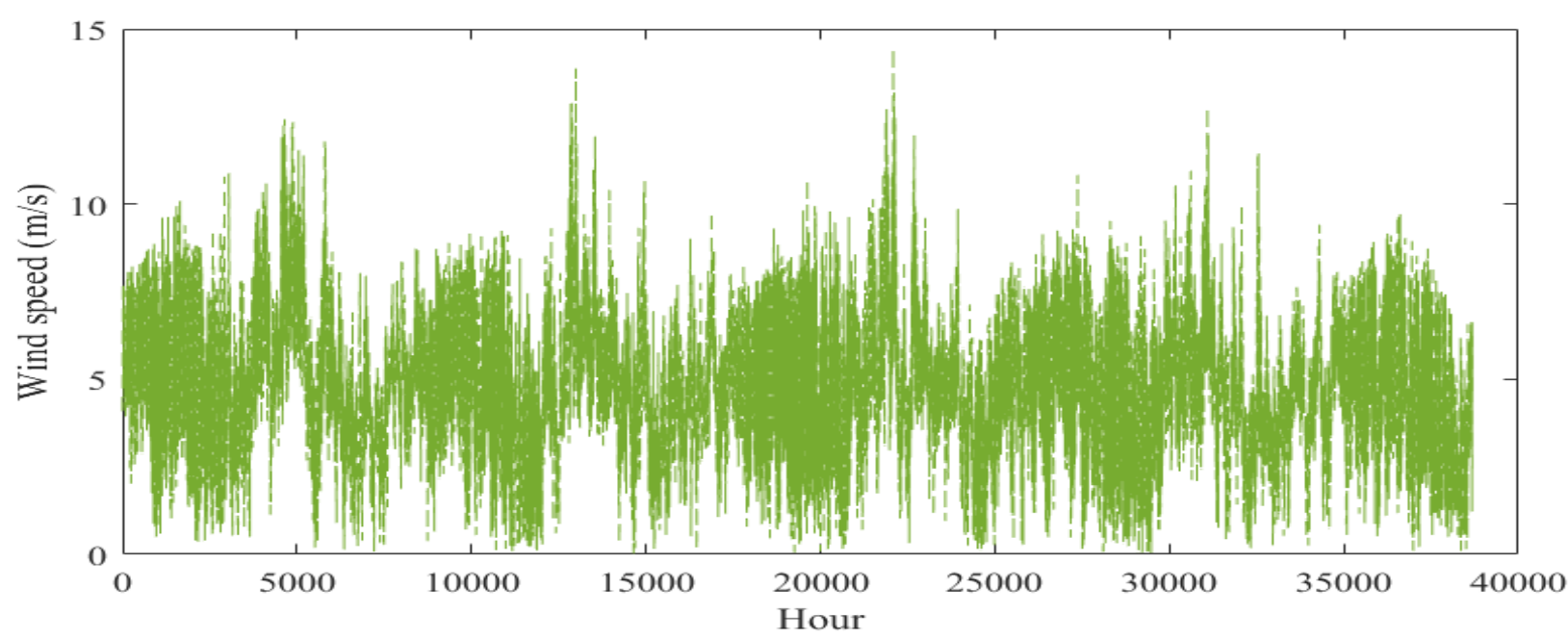

294

(c) Salem Station 


$$
\circ \mathrm{MASE}=\text { mean }|z| \text {; where } z=\frac{f}{\frac{\sum_{k=2}^{N}\left|o_{i}-o_{i-1}\right|}{N-1}}, f \text { is the forecast error in the given period. }
$$

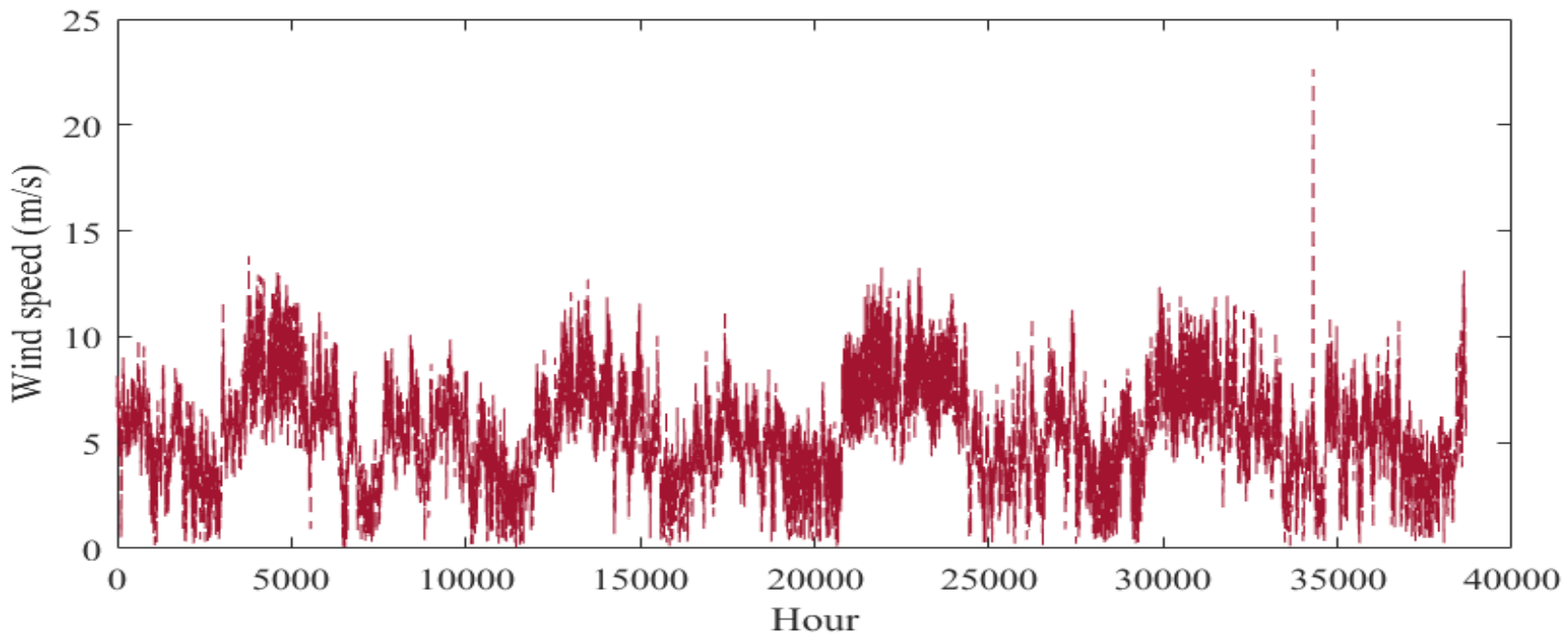

(d) Tirunelveli Station

Figure 2. Hourly wind speed data measured during January 2014 to May 2018

\section{Experimental setup and numerical analysis}

To illustrate the prediction capability of the proposed MKLSTSVR and MHKLSTSVR models their performances on a few WS data are compared with TSVR, PLSTSVR and LDMR. The performances of these implemented models are evaluated using RMSE (Root Mean Squared Error), MAE (Mean Absolute Error), SMAPE (Symmetric Mean Absolute Percentage Error), MASE (Mean Absolute Scaled Error)and $\mathrm{R}^{2}$ (Coefficient of correlation). The definitions of these evaluators can beexpressed as:

○ $\mathrm{RMSE}=\sqrt{\frac{\sum_{k=1}^{N}(o-p)}{N},}$

$\circ \quad \mathrm{MAE}=\frac{\sum_{k=1}^{N}|o-p|}{N}$,

○ SMAPE $=\frac{\sum_{k=1}^{N}|o-p|}{N(p+o)}$,

Here, $o$ and $p$ indicates the observed and the predicted WS values respectively. 
All the experiments are conducted on Window 8 installed laptop computer with 4 GB RAM. The QPPs of TSVR and LDMR models are solved using the external MOSEK optimization toolbox. The Gaussian kernel is used for the TSVR, PLSTSVR and LDMR models. The Gaussian kernel may be represented as:

$$
K\left(d_{m}, d_{n}\right)=\exp \left(-\mu\left\|d_{m}-d_{n}\right\|^{2}\right), \text { for } m, n=1, \ldots, m,
$$

where $d_{m}, d_{n}$ indicates the input data samples. The Gaussian kernel parameter $\mu>0$ is selected from the range $\left\{2^{-5}, \ldots, 2^{5}\right\}$ for TSVR, PLSTSVR and LDMR models. The value of the $\varepsilon$ parameter is set from $\varepsilon=\{0.05,0.1,0.5,1,1.5,2\}$ for all models including the proposed MKLSTSVR and MHKLSTSVR. The model parameters $C_{1}=C_{2}$ are considered from $\left\{10^{-5}, 10^{-3}, \ldots, 10^{5}\right\}$ for all models. The Gaussian kernel parameter $\lambda$ is taken from the set $\left\{2^{-5}, 2^{-3}, \ldots, 2^{5}\right\}$ for TSVR, PLSTSVR and LDMR models. The $\lambda$ values are chosen from $\left\{10^{-5}, 10^{-3}, . ., 10^{5}\right\}$ for LDMR. For the proposed MKLSTSVR and MHKLSTSVR models, the wavelet kernel parameter $d$ is fixed to 1 .

Two different lag periods are used namely 1-3 and 1-5. Table 4and Table 5portray the results based on the various performance evaluatorsused in this study for the various cities considered for 1-3 and 1-5 respectively. From Table 4, the following conclusionscan be derived:

a) The proposed MKLSTSVR and MHKLSTSVR show the best performance based on RMSE in 0 and 2 cases out of 4 respectively.

b) In the case of MAE, the proposed MKLSTSVR and MHKLSTSVR obtains best results in 1 case each.

c) The proposed MKLSTSVR and MHKLSTSVR reportthebest results based on SMAPE in 2 and 1 cases respectively.

d) The proposed MKLSTSVR and MHKLSTSVR indicatesthebest performance based on MASE in 1 and 1 case out of 4 respectively.

e) The proposed MKLSTSVR and MHKLSTSVR show the best performance based on $\mathrm{R}^{2}$ in 0 and 2 cases out of 4 respectively.

Table 4. Comparison of TSVR, PLSTSVR, LDMR, MKLSTSVR and MHKLSTSVR based on the performance evaluators, optimum parameters and the computational times for 1-3 lag time period. (Best results are bolded) 


\begin{tabular}{|c|c|c|c|c|c|c|}
\hline $\begin{array}{l}\text { Dataset } \\
\text { (train, test) }\end{array}$ & $\begin{array}{c}\text { Performance } \\
\text { Evaluators }\end{array}$ & $\begin{array}{c}\text { TSVR } \\
(C, \mu, \varepsilon) \\
\text { time (sec) }\end{array}$ & $\begin{array}{l}\text { PLSTSVR } \\
(C, \mu, \varepsilon) \\
\text { time (sec) }\end{array}$ & $\begin{array}{c}\text { LDMR } \\
\left(C_{1}, \mu, C_{2}, \varepsilon\right) \\
\text { time (sec) }\end{array}$ & $\begin{array}{l}\text { MKLSTSVR } \\
\qquad(C, \varepsilon) \\
\text { time (sec) }\end{array}$ & $\begin{array}{l}\text { MHKLSTSVR } \\
\qquad\left(\begin{array}{l}C \\
\text { time }(\mathrm{sec})\end{array}\right.\end{array}$ \\
\hline \multirow{7}{*}{ Chennai } & RMSE & 0.040317 & 0.032152 & 0.031793 & 0.031951 & 0.031890 \\
\hline & MAE & 0.030705 & 0.023787 & 0.023889 & 0.023762 & 0.024037 \\
\hline & SMAPE & 0.110742 & 0.090936 & 0.091312 & 0.091740 & 0.095099 \\
\hline & MASE & 0.943912 & 0.731252 & 0.734401 & 0.730474 & 0.738916 \\
\hline & $\mathrm{R}^{2}$ & 0.91958 & 0.94218 & 0.9434 & 0.942841 & 0.942999 \\
\hline & Parameters & $\left(10^{\wedge}-3,8,1.5\right)$ & $\left(10^{\wedge}-5,4,1\right)$ & $\left(10^{\wedge}-1,8,0.001,0.05\right)$ & $\left(10^{\wedge}-5,0.5\right)$ & $\left(10^{\wedge}-5,1.5\right)$ \\
\hline & Time & 9.77998 & 1.18909 & 1.09127 & 0.992544 & 0.861059 \\
\hline \multirow{7}{*}{ Madurai } & RMSE & 0.065534 & 0.05581 & 0.053725 & 0.054514 & 0.055597 \\
\hline & MAE & 0.049161 & 0.041094 & 0.040596 & 0.040875 & 0.041337 \\
\hline & SMAPE & 0.183424 & 0.154061 & 0.152974 & 0.153802 & 0.156680 \\
\hline & MASE & 0.959875 & 0.802353 & 0.79264 & 0.798084 & 0.807099 \\
\hline & $\mathrm{R}^{2}$ & 0.79137 & 0.84623 & 0.85563 & 0.851104 & 0.845275 \\
\hline & Parameters & $\left(10^{\wedge}-1,8,0.05\right)$ & $\left(10^{\wedge}-5,8,1\right)$ & $\left(10^{\wedge}-3,8,0.001,1\right)$ & $\left(10^{\wedge}-5,2\right)$ & $\left(10^{\wedge}-5,2\right)$ \\
\hline & Time & 5.44994 & 1.52731 & 1.15297 & 0.96967 & 0.887909 \\
\hline \multirow{7}{*}{ Salem } & RMSE & 0.066159 & 0.056481 & 0.058038 & 0.055535 & 0.055013 \\
\hline & MAE & 0.053527 & 0.041053 & 0.041513 & 0.040799 & 0.040412 \\
\hline & SMAPE & 0.196464 & 0.154028 & 0.156257 & 0.153872 & 0.155207 \\
\hline & MASE & 1.04695 & 0.802962 & 0.811969 & 0.798012 & 0.790428 \\
\hline & $\mathrm{R}^{2}$ & 0.82664 & 0.84468 & 0.83438 & 0.848699 & 0.85122 \\
\hline & Parameters & $\left(10^{\wedge}-1,2,0.05\right)$ & $\left(10^{\wedge}-5,2,1.5\right)$ & $\left(10^{\wedge}-3,8,0.001,0.1\right)$ & $\left(10^{\wedge}-5,2\right)$ & $\left(10^{\wedge}-5,2\right)$ \\
\hline & Time & 9.36352 & 1.64833 & 1.15626 & 1.01518 & 0.879462 \\
\hline \multirow{7}{*}{ Tirunelveli } & RMSE & 0.037187 & 0.029061 & 0.029063 & 0.029685 & 0.290056 \\
\hline & MAE & 0.027843 & 0.0215 & 0.021501 & 0.021537 & 0.021516 \\
\hline & SMAPE & 0.131682 & 0.112339 & 0.11234 & 0.112068 & 0.111240 \\
\hline & MASE & 1.05238 & 0.812639 & 0.812653 & 0.814044 & 0.813238 \\
\hline & $\mathrm{R}^{2}$ & 0.90645 & 0.93187 & 0.93186 & 0.928904 & 0.932099 \\
\hline & Parameters & $\left(10^{\wedge}-1,2,2\right)$ & $\left(10^{\wedge}-5,2,2\right)$ & $\left(10^{\wedge} 0,2,0.00001,0.5\right)$ & $\left(10^{\wedge}-5,0.1\right)$ & $\left(10^{\wedge}-5,2\right)$ \\
\hline & Time & 9.31972 & 1.22065 & 1.11234 & 0.927693 & 0.893671 \\
\hline
\end{tabular}

Further, the size of the window is increased from 1-3 to 1-5 to enhance the performance.

350 In Table 5, the results are tabulated using a lag window of 5.From Table 5, the following 351 conclusions can be derived:

352 a) The proposed MKLSTSVR and MHKLSTSVR reveal the best results based on RMSE in 3532 cases each out of 4 respectively.

354 b) The proposed MKLSTSVR and MHKLSTSVR reportthebest performance based on MAE in 2 cases each. 
356 c) Based on SMAPE, the proposed MKLSTSVR and MHKLSTSVR show the best 357 performance in 3 and 1 case out of 4 respectively.

358 d) The best results are noticeable in 2 and 2 cases out of 4 cases for the proposed $359 \quad$ MKLSTSVR and MHKLSTSVR respectively, based on MASE.

360 e) For $\mathrm{R}^{2}$, the proposed MKLSTSVR and MHKLSTSVR portray the best results in 1 and 3 $361 \quad$ cases out of 4 respectively.

362 Table 5:Comparison of TSVR, PLSTSVR, LDMR, MKLSTSVR and MHKLSTSVR based on the performance evaluators, optimum parameters

363 and the computational times for 1-5 lag time period. (Best results are bolded)

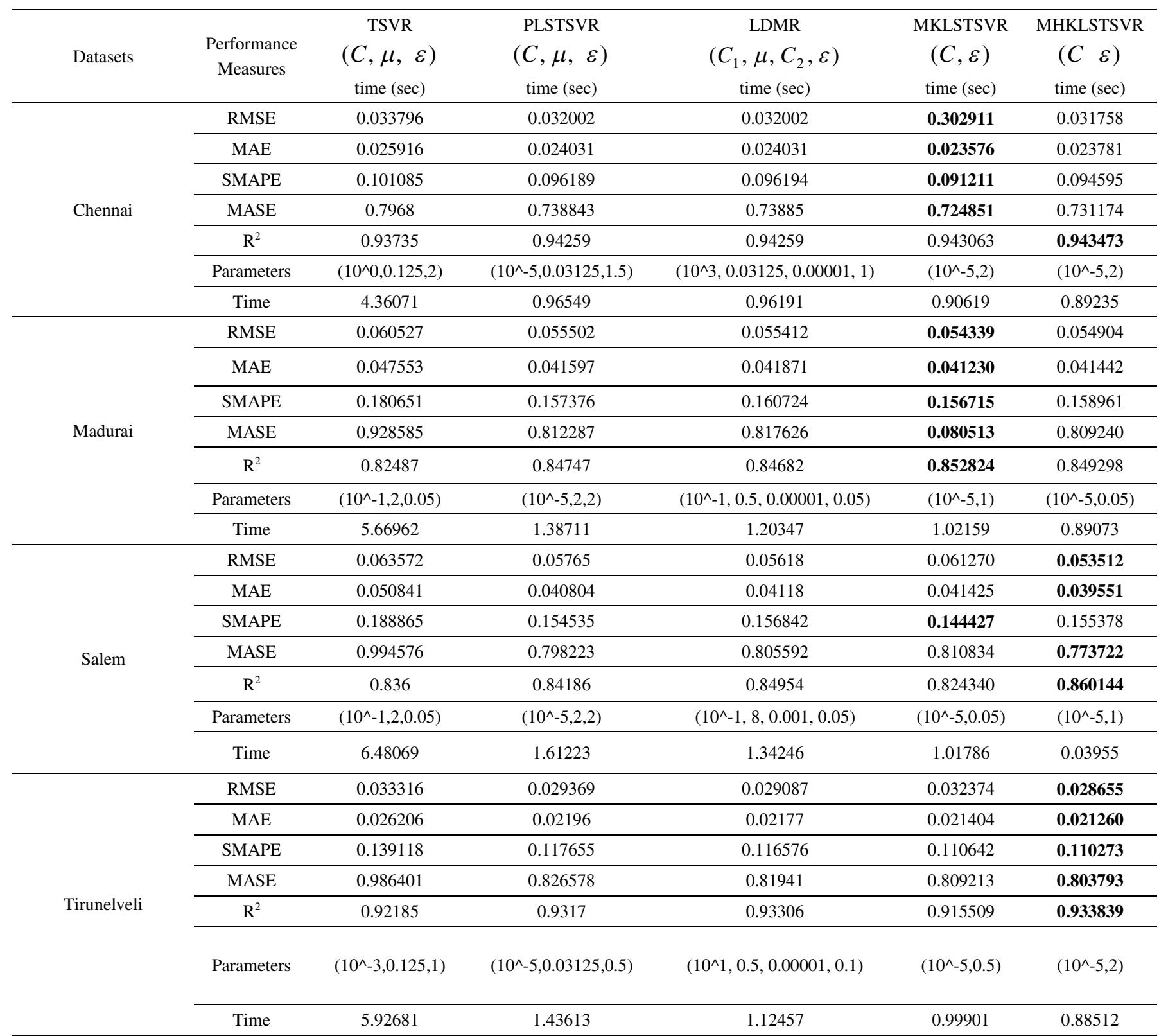


Overall, it is noticeable that the increase in the time lag period shows improved performance for the proposed MKLSTSVR and MHKLSTSVR.Figures 3-6 show the comparison between the

367

373

374

375

376

377

378

379

380

381

382

383

384

385

386

387

388

389

390

391

392

393

394 actual and predicted WS values of TSVR, PLSTSVR, LDMR and the proposed MKLSTSVR and MHKLSTSVR for 1-5. Although all the plots seem to be similar, the overall performances of the proposed models are better compared toTSVR, PLSTSVR and LDMR models based on the evaluators.
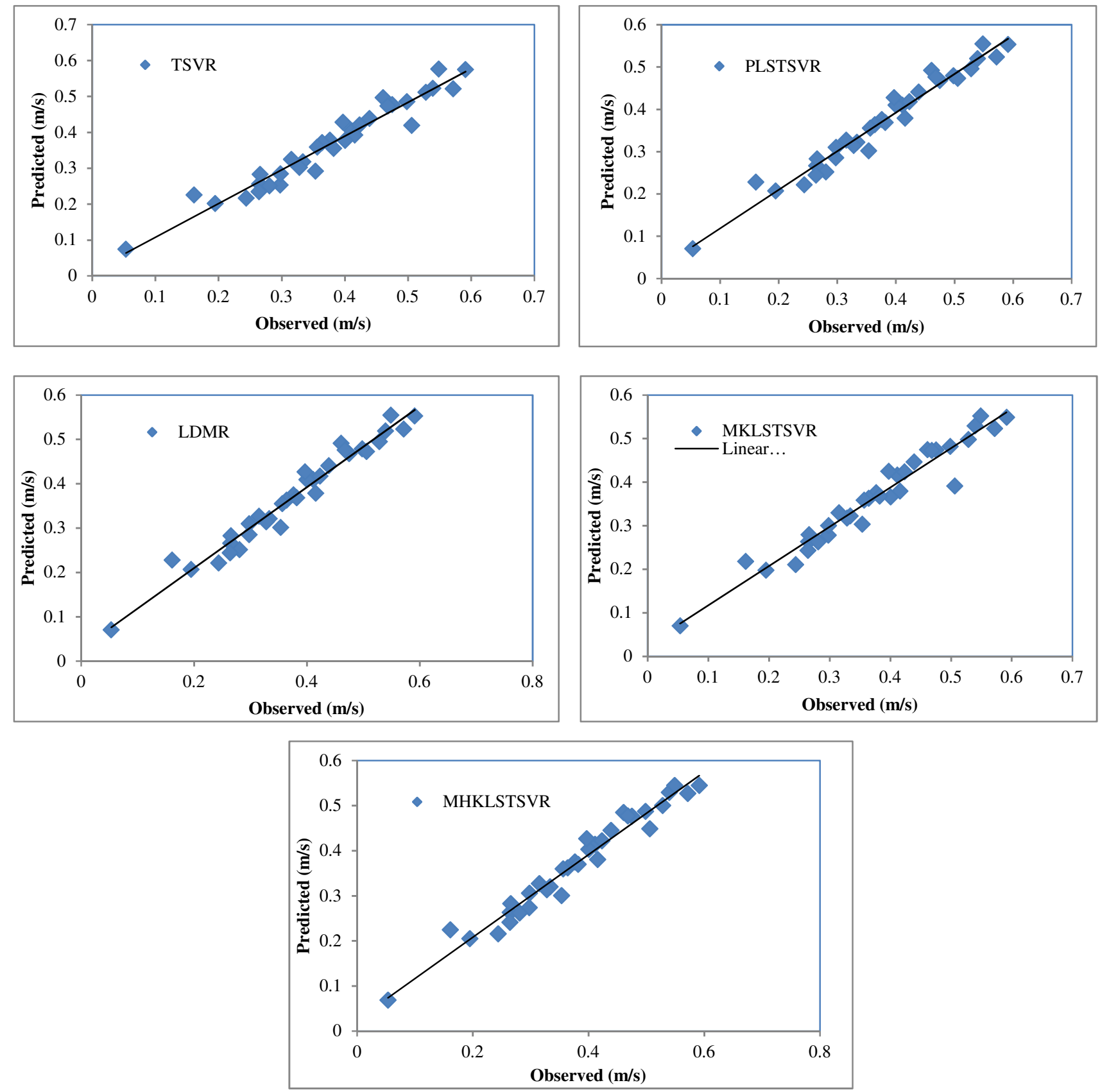

Figure 3. Comparison of observed and predicted WS values for Chennai using a lag window of 5. 

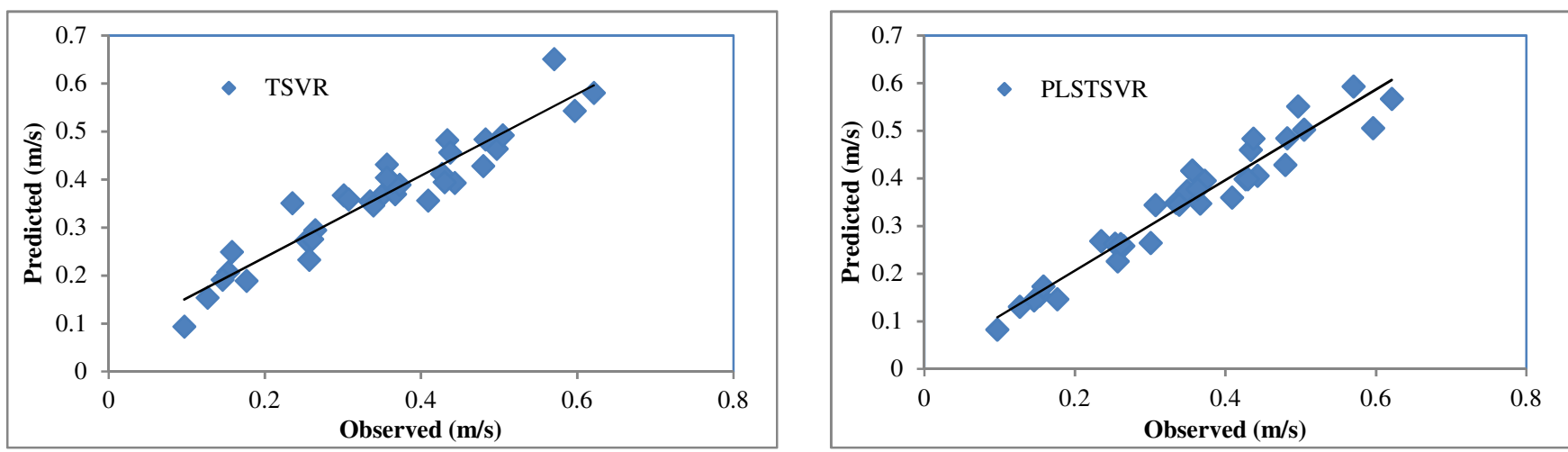

402

403

404

405

406

407

408
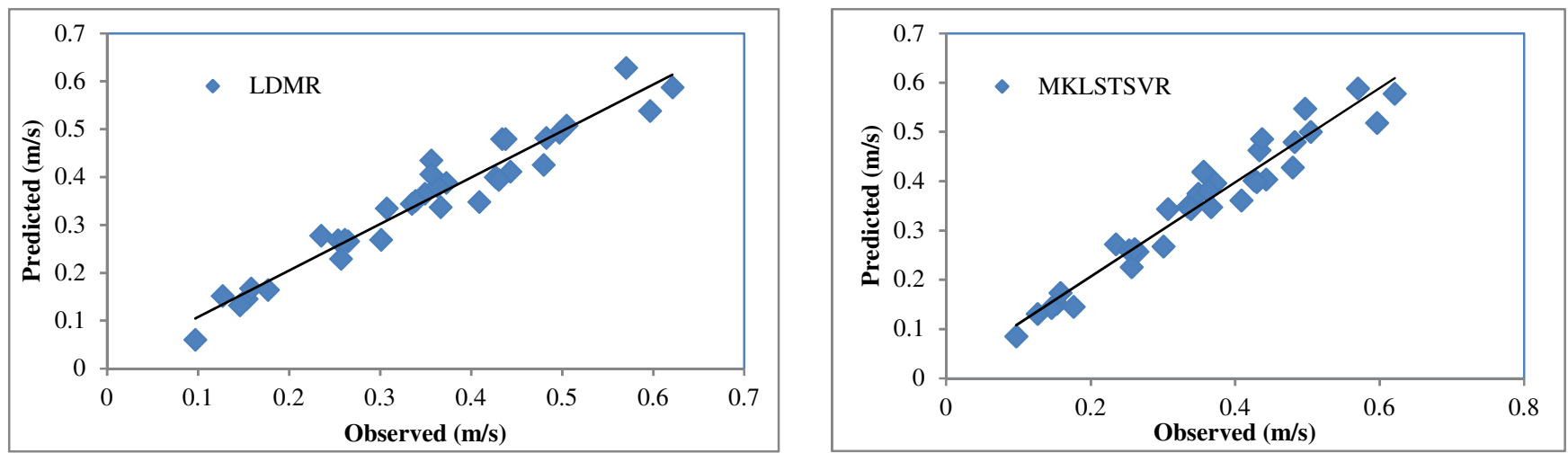

409

410

411

412

413

414

415

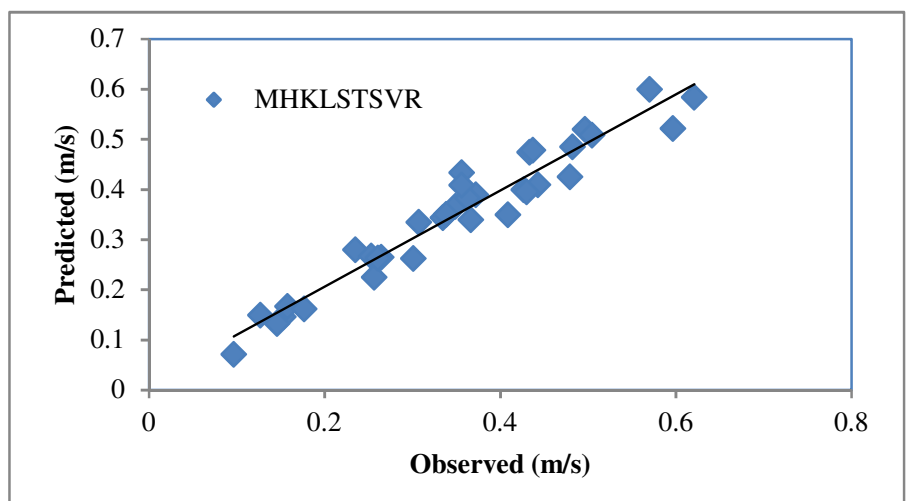

416

Figure 4. Comparison of observed and predicted WS values for Madurai using a lag window of 5.

417 It can also be observed from Table 4 and Table 5 shows that the proposedMKLSTSVR and

418 MHKLSTSVR take less time for computation compared to other models. Thus, it will be

419 interesting to seehow the proposed MKLSTSVR and MHKLSTSVR behave in short term WS

420 prediction at different sites.

421
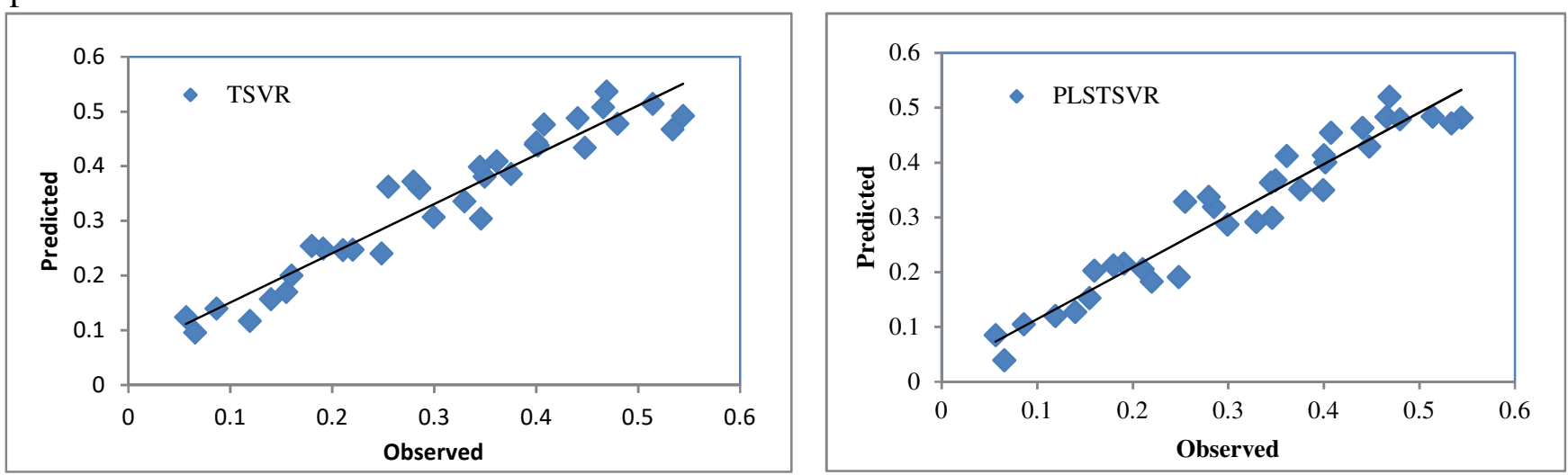

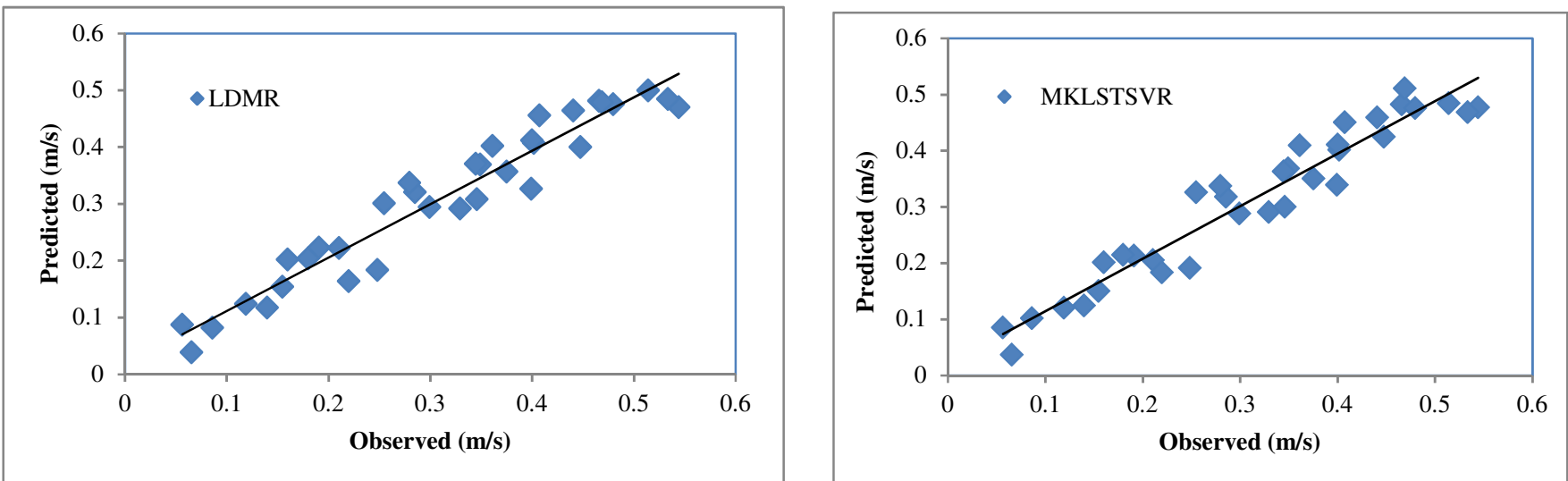

432

433

434

435

436

437

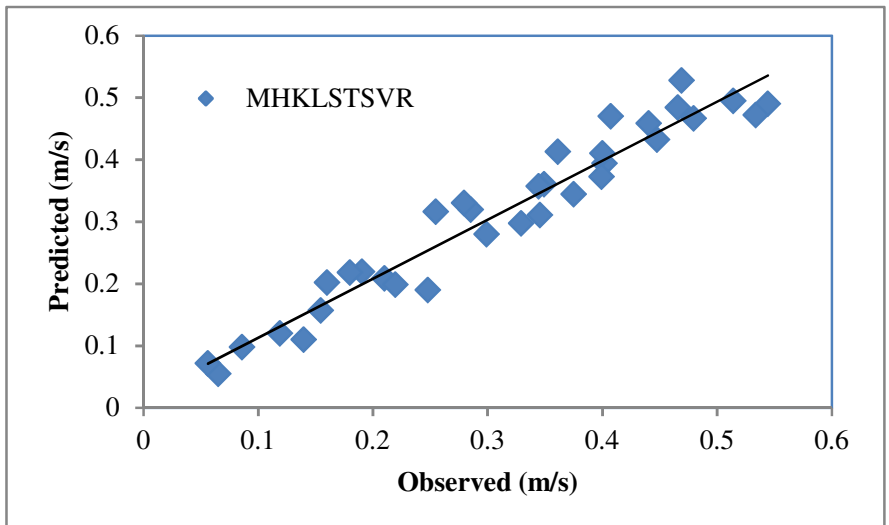

438

Figure 5. Comparison of observed and predicted WS values for Salem using a lag window of 5.

439

440

441

442

443

444

445
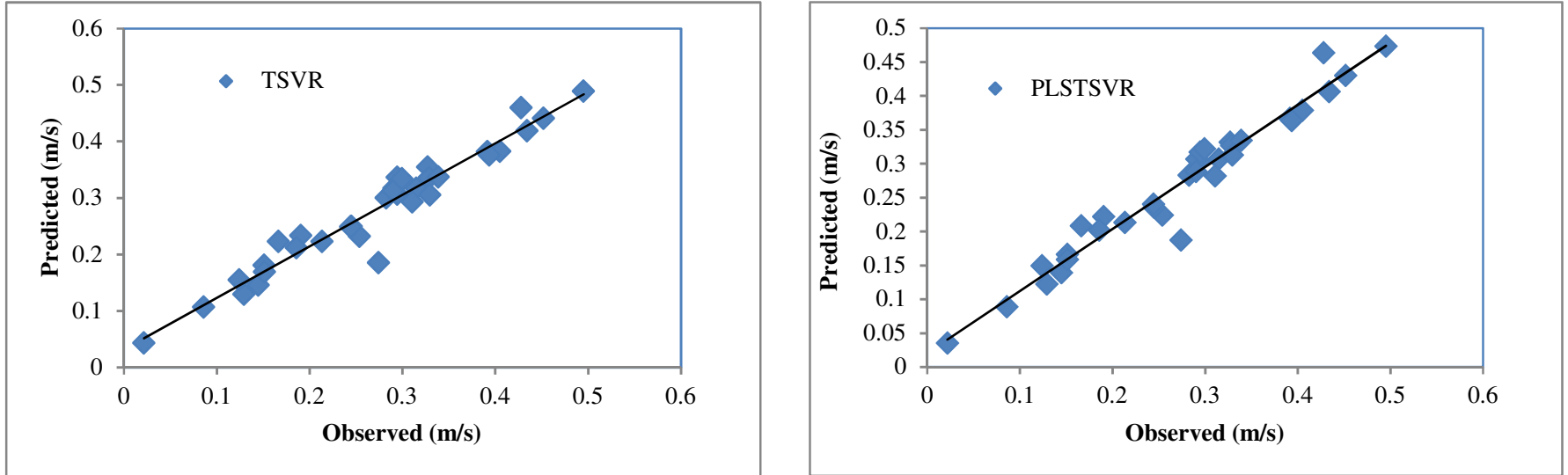

446

447

448

449

450

451
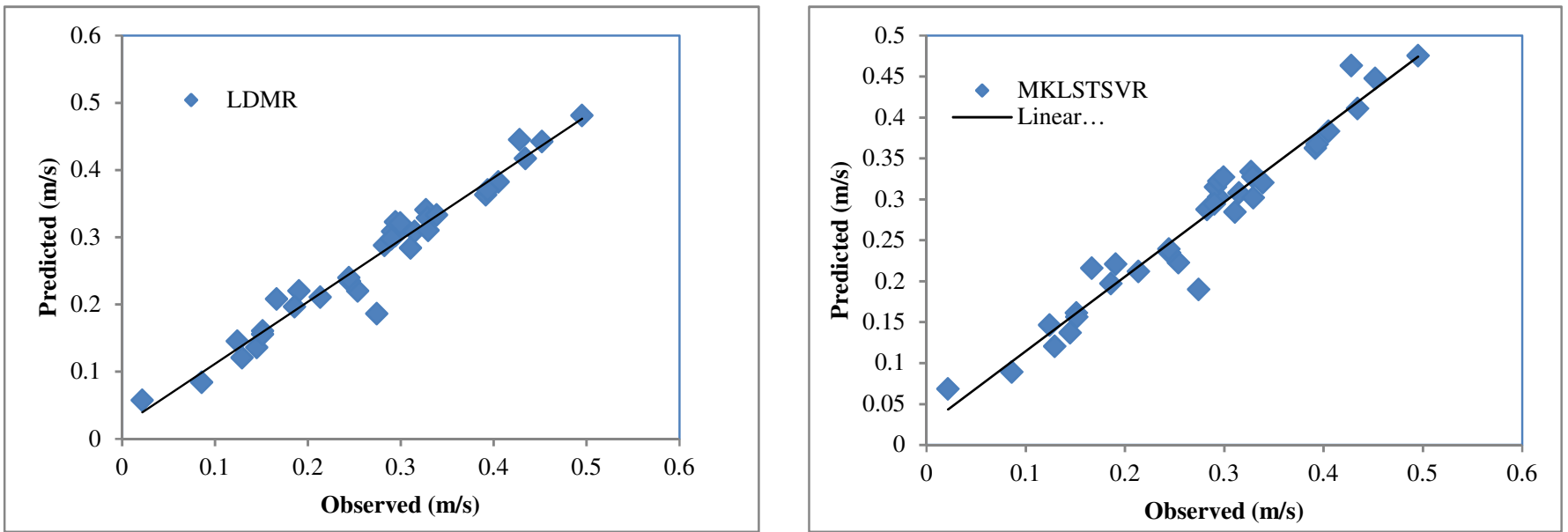


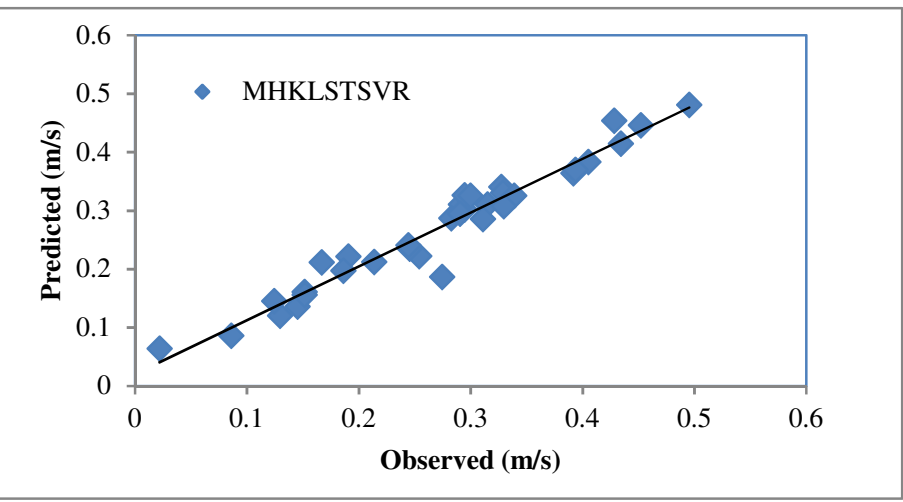

Figure 6. Comparison of observed and predicted WS values for Tirunelveli using a lag window of 5.

458

459

460

461

462

463

464

465

466

467

468

469

470

471

472

473

474

475

476

477

478

479

480

\section{Conclusion}

The wavelets are powerful enough to deal with the non-stationary datasets. Moreover, the PLSTSVR model shows excellent prediction performance. Hence in this work, the wavelet kernels are embedded in the PLSTSVR models and two novel wavelets kernel-based PLSTSVR models namely, MKLSTSVR and MHKLSTSVR are proposed for WS prediction in four different wind-stations in India. The resultsshow that the proposed models to be superior when compared to the related models: i.e., TSVR, PLSTSVR and LDMR. Amongthe proposed MKLSTSVR and MHKLSTSVR models, the latter shows a closer relationship with the original data. Moreover, the proposed models are computationally efficient as they take less time for computation compared to the other reported models. Overall, one can conclude that both MKLSTSVR and MHKLSTSVR are efficient modelsand applicable for short term WS prediction. These models can also be applied in the field of engineering like a prediction of river suspended sediment load, rainfall forecasting, runoff prediction, etc.

Availability of data and materials: The datasets that has been used in this study are available from co-author on reasonable request.

Authors Contribution: Role of Barenya Bikash Hazarika- Conceptualization, methodology, writing original draft and visualization. Role of Deepak Gupta- Investigation, validation, writing review and editing. Role of Narayanan Natarajan- Writing original draft and formal analysis.

Funding: Not applicable

\section{Declarations}

Ethics approval: Not applicable

Concent to participate: Not applicable

Consent to publish: Not applicable. 


\section{References}

Bakhsh, H., Srinivasan, R., \&Bahel, V. (1985). Correlation between hourly diffuse and global radiation for Dhahran, Saudi Arabia. Solar \& Wind Technology, 2(1), 59-61.

Blanchard, T., \&Samanta, B. (2020). Wind speed forecasting using neural networks. Wind Engineering, 44(1), 33-48.

Daubechies, I. (1990a). The wavelet transform, time-frequency localization and signal analysis. IEEE transactions on information theory, 36(5), 961-1005.

Daubechies, I. (1992b). Ten lectures on wavelets. Society for industrial and applied mathematics.

Dhiman, H. S., Anand, P., \& Deb, D. (2019). Wavelet transform and variants of svr with application in wind forecasting. In Innovations in Infrastructure (pp. 501-511). Springer, Singapore.

Ding, S., Wu, F., \& Shi, Z. (2014). Wavelet twin support vector machine. Neural Computing and Applications, 25(6), 1241-1247.

Ding, S., Zhang, J., Xu, X., \& Zhang, Y. (2016). A wavelet extreme learning machine. Neural Computing and Applications, 27(4), $1033-1040$.

Fu, C., Li, G. Q., Lin, K. P., \& Zhang, H. J. (2019). Short-term wind power prediction based on improved chicken algorithm optimization support vector machine. Sustainability, 11(2), 512.

Gupta, D. (2017). Training primal K-nearest neighbor based weighted twin support vector regression via unconstrained convex minimization. Applied Intelligence, 47(3), 962-991.

Harbola, S., \& Coors, V. (2019). One dimensional convolutional neural network architectures for wind prediction. Energy Conversion and Management, 195, 70-75.

Hazarika, B. B., \& Gupta, D. (2020). Modelling and forecasting of COVID-19 spread using wavelet-coupled random vector functional link networks. Applied Soft Computing, 96, 106626.

Hazarika, B. B., Gupta, D., \& Berlin, M. (2020a). A coiflet LDMR and coiflet OB-ELM for river suspended sediment load prediction. International Journal of Environmental Science and Technology, 1-18.

Hazarika, B. B., Gupta, D., \& Berlin, M. (2020b). Modeling suspended sediment load in a river using extreme learning machine and twin support vector regression with wavelet conjunction. Environmental Earth Sciences, 79, 234.

He, J., \& Xu, J. (2019). Ultra-short-term wind speed forecasting based on support vector machine with combined kernel function and similar data. EURASIP Journal on Wireless Communications and Networking, 2019(1), 248.

Holland, J. H. (1992). Adaptation in natural and artificial systems: an introductory analysis with applications to biology, control, and artificial intelligence. MIT press.

Huang, H. J., Ding, S. F., \& Shi, Z. Z. (2013). Primal least squares twin support vector regression. Journal of Zhejiang University SCIENCE $C, 14(9), 722-732$.

Jamil, M., \& Zeeshan, M. (2019). A comparative analysis of ANN and chaotic approach-based wind speed prediction in India. Neural Computing and Applications, 31(10), 6807-6819.

Jamil, M., \& Zeeshan, M. (2019). A comparative analysis of ANN and chaotic approach-based wind speed prediction in India. Neural Computing and Applications, 31(10), 6807-6819.

Jha, S. K., \&Bilalovikj, J. (2019). Short-term wind speed prediction at Bogdanci power plant in FYROM using an artificial neural network. International Journal of Sustainable Energy, 38(6), 526-541.

Khosravi, A., Koury, R. N. N., Machado, L., \& Pabon, J. J. G. (2018). Prediction of wind speed and wind direction using artificial neural network, support vector regression and adaptive neuro-fuzzy inference system. Sustainable Energy Technologies and Assessments, 25, 146-160. Li, L. L., Zhao, X., Tseng, M. L., \& Tan, R. R. (2020). Short-term wind power forecasting based on support vector machine with improved dragonfly algorithm. Journal of Cleaner Production, 242, 118447.

Liu, D., Niu, D., Wang, H., \& Fan, L. (2014). Short-term wind speed forecasting using wavelet transform and support vector machines optimized by genetic algorithm. Renewable Energy, 62, 592-597.

Liu, M., Cao, Z., Zhang, J., Wang, L., Huang, C., \& Luo, X. (2020). Short-term wind speed forecasting based on the Jaya-SVM model. International Journal of Electrical Power \& Energy Systems, 121, 106056.

Liu, Y., Shi, J., Yang, Y., \& Lee, W. J. (2012). Short-term wind-power prediction based on wavelet transform-support vector machine and statistic-characteristics analysis. IEEE Transactions on Industry Applications, 48(4), 1136-1141.

Mallat, S. (1999). A wavelet tour of signal processing. Elsevier.

Mi, X., Liu, H., \& Li, Y. (2019). Wind speed prediction model using singular spectrum analysis, empirical mode decomposition and convolutional support vector machine. Energy Conversion and Management, 180, 196-205.

Morlet, J., Arens, G., Fourgeau, E., \&Giard, D. (1982a). Wave propagation and sampling theory—Part II: Sampling theory and complex waves. Geophysics, 47(2), 222-236.

Morlet, J., Arens, G., Fourgeau, E., \&Glard, D. (1982b). Wave propagation and sampling theory-Part I: Complex signal and scattering in multilayered media. Geophysics, 47(2), 203-221.

Natarajan, Y. J., \&Nachimuthu, D. S. (2019). New SVM kernel soft computing models for wind speed prediction in renewable energy applications. Soft Computing, 1-18.

Peng, X. (2010). TSVR: an efficient twin support vector machine for regression. Neural Networks, 23(3), 365-372. 
Prasetyowati, A., Sudiana, D., \&Sudibyo, H. (2019, October). Prediction of Wind Power Model Using Hybrid Method Based on WD-SVM Algorithm: Case Study Pandansimo Wind Farm. In Journal of Physics: Conference Series (Vol. 1338, No. 1, p. 012048). IOP Publishing. Qolipour, M., Mostafaeipour, A., Saidi-Mehrabad, M., \&Arabnia, H. R. (2019). Prediction of wind speed using a new grey-extreme learning machine hybrid algorithm: A case study. Energy \& Environment, 30(1), 44-62.

Rastogi, R., Anand, P., \& Chandra, S. (2020). Large-margin distribution machine-based regression. Neural Computing and Applications, 32(8), 3633-3648. ensemble approach for wind speed prediction. Neural Computing and Applications, 33(7), 2369-2391.

Salcedo-Sanz, S., Ortiz-Garc1, E. G., Pérez-Bellido, Á. M., Portilla-Figueras, A., \& Prieto, L. (2011). Short term wind speed prediction based on evolutionary support vector regression algorithms. Expert Systems with Applications, 38(4), 4052-4057.

SangitaB, P., \& Deshmukh, S. R. (2011, December). Use of Support Vector Machine, decision tree and Naive Bayesian techniques for wind speed classification. In 2011 International Conference on Power and Energy Systems (pp. 1-8). IEEE.

Sifuzzaman, M., Islam, M. R., \& Ali, M. Z. (2009). Application of Wavelet Transform and its Advantages Compared to Fourier Transform. Journal of Physical Sciences, 13, 121-134.

Sivanagaraja, T., Tatinati, A. K., \&Veluvolu, K. C. A Hybrid Method based on Discrete Wavelets and Least Squares Support Vector Machines for Short-Term Wind Speed Forecasting.

Sun, Y., Li, L. L., Huang, X. S., \& Duan, C. Y. (2013). Short-term wind speed forecasting based on optimizated support vector machine. In Applied Mechanics and Materials (Vol. 300, pp. 189-194). Trans Tech Publications Ltd.

Tian, Z. (2020). Short-term wind speed prediction based on LMD and improved FA optimized combined kernel function LSSVM. Engineering Applications of Artificial Intelligence, 91, 103573.

Tian, Z., Ren, Y., \& Wang, G. (2019). Short-term wind speed prediction based on improved PSO algorithm optimized EM-ELM. Energy Sources, Part A: Recovery, Utilization, and Environmental Effects, 41(1), 26-46.

Vinothkumar, T., \&Deeba, K. (2020). Hybrid wind speed prediction model based on recurrent long short-term memory neural network and support vector machine models. Soft Computing, 24(7), 5345-5355.

Wang, C., Wu, J., Wang, J., \& Hu, Z. (2016). Short-term wind speed forecasting using the data processing approach and the support vector machine model optimized by the improved cuckoo search parameter estimation algorithm. Mathematical Problems in Engineering, 2016.

Wang, J., \& Yang, Z. (2021). Ultra-short-term wind speed forecasting using an optimized artificial intelligence algorithm. Renewable Energy, 171, 1418-1435.

Wang, J., Zhou, Q., Jiang, H., \& Hou, R. (2015). Short-term wind speed forecasting using support vector regression optimized by cuckoo optimization algorithm. Mathematical Problems in Engineering, 2015.

Wu, Q., \& Lin, H. (2019). Short-term wind speed forecasting based on hybrid variational mode decomposition and least squares support vector machine optimized by bat algorithm model. Sustainability, 11(3), 652.

Xiang, L., Deng, Z., \& Hu, A. (2019). Forecasting short-term wind speed based on IEWT-LSSVM model optimized by bird swarm algorithm. IEEE Access, 7, 59333-59345.

Xiao, L., Shao, W., Jin, F., \& Wu, Z. (2021). A self-adaptive kernel extreme learning machine for short-term wind speed forecasting. Applied Soft Computing, 99, 106917.

Zeng, J., \&Qiao, W. (2012). Short-term wind power prediction using a wavelet support vector machine. IEEE transactions on sustainable energy, 3(2), 255-264.

Zhang, L., Zhou, W., \& Jiao, L. (2004). Wavelet support vector machine. IEEE Transactions on Systems, Man, and Cybernetics, Part B (Cybernetics), 34(1), 34-39.

Zhang, T., \& Zhou, Z. H. (2014, August). Large margin distribution machine. In Proceedings of the 20th ACM SIGKDD international conference on Knowledge discovery and data mining (pp. 313-322).

Zhang, Y., Pan, G., Chen, B., Han, J., Zhao, Y., \& Zhang, C. (2020). Short-term wind speed prediction model based on GA-ANN improved by VMD. Renewable Energy, 156, 1373-1388.

Zhang, Z., Ye, L., Qin, H., Liu, Y., Wang, C., Yu, X., .. \& Li, J. (2019). Wind speed prediction method using shared weight long short-term memory network and Gaussian process regression. Applied energy, 247, 270-284.

Zhou, X. Y., \& Ye, Y. Z. (2006). Application of wavelet analysis to fault diagnosis. Control Engineering of China, 13(1), 70-73. 